Research Paper

\title{
Serum Lactate Dehydrogenase Levels as a Predictive Marker of Oxaliplatin-Induced Hypersensitivity Reactions in Japanese Patients with Advanced Colorectal Cancer
}

Kyoko Seki 1, Yasuo Tsuduki 2, Takeshi Ioroi 3, Michiko Yamane 4, Hiroko Yamauchi 5, Yukinari Shiraishi 1, Tadaaki Ogawa 1, Izumi Nakata 2, Kohshi Nishiguchi ${ }^{3}$, Teruhisa Matsubayashi ${ }^{4}$, Yoshihide Takakubo ${ }^{5}$, Motohiro Yamamori 6, Akiko Kuwahara ${ }^{6}$, Noboru Okamura ${ }^{6}$, and Toshiyuki Sakaeda $3,7,8 \bowtie$

1. Department of Pharmacy, Japan Labour Health and Welfare Organization, Kobe Rosai Hospital, Kobe 651-0053, Japan

2. Department of Pharmacy, National Hospital Organization Kobe Medical Center, Kobe 654-0155, Japan

3. Department of Pharmacy, Kobe University Hospital, Kobe 650-0017, Japan

4. Department of Pharmacy, Japanese Red Cross Kobe Hospital, Kobe 651-0073, Japan

5. Department of Pharmacy, Shinko Hospital, Kobe 651-0072, Japan

6. School of Pharmacy and Pharmaceutical Sciences, Mukogawa Women's University, Nishinomiya 663-8179, Japan

7. Graduate School of Pharmaceutical Sciences, Kyoto University, Kyoto 606-8501, Japan

8. Department of Pharmacokinetics, Kyoto Pharmaceutical University, Kyoto 607-8414, Japan

$\square$ Corresponding author: Toshiyuki Sakaeda, Ph.D., Department of Pharmacokinetics, Kyoto Pharmaceutical University, Kyoto 607-8414, Japan. Tel: +81-75-595-4625, Fax: +81-75-595-4751, E-mail: sakaedat@mb.kyoto-phu.ac.jp

(c) Ivyspring International Publisher. This is an open-access article distributed under the terms of the Creative Commons License (http://creativecommons.org/ licenses/by-nc-nd/3.0/). Reproduction is permitted for personal, noncommercial use, provided that the article is in whole, unmodified, and properly cited.

Received: 2013.09.12; Accepted: 2014.03.12; Published: 2014.04.26

\begin{abstract}
Objective: Clinical laboratory test data obtained prior to treatments were previously analyzed from the standpoint of susceptibility to hypersensitivity reactions in patients treated with the platimun anticancer agent, oxaliplatin (L-OHP). In the present study, the time course from the first to last cycle of the treatment was additionally analyzed to determine a better predictor of these reactions.

Methods: A total of 20 laboratory test data were obtained from 108 Japanese patients with advanced colorectal cancer who were treated with the L-OHP-containing regimens, FOLFOX4 and/or mFOLFOX6. The averages and variation coefficients (CV\%) of the data until the last cycle of the treatment were compared between patients with hypersensitivity reactions and those without.

Results: The average serum lactate dehydrogenase (LDH) level was lower in patients with grade $1 / 2$ reactions $(P=0.016)$, whereas its $C V \%$ value was higher in patients with grade $3 / 4$ reactions $(P=0.005)$ than in those without reactions. An increase in serum LDH levels was observed in some patients with grade $3 / 4$ reactions as the cycle number increased, and thereafter hypersensitivity reactions occurred. This phenomenon was not always observed, but was never detected in patients with grade $\mathrm{I} / 2$ reactions.

Conclusions: Serum LDH levels may be a predictive marker of hypersensitivity reactions in patients treated with L-OHP. Further extensive examinations with a larger number of patients are needed to establish a patient management strategy.
\end{abstract}

Key words: colorectal cancer, FOLFOX, oxaliplatin, hypersensitivity reactions, serum lactate dehydrogenase level 


\section{Introduction}

Oxaliplatin (L-OHP) is a third-generation platinum anticancer drug, and L-OHP-containing regimens, including FOLFOX4 and mFOLFOX6, are currently standard treatments for colorectal cancer [1-5]. The dose-limiting toxicities of L-OHP were shown to be cumulative sensory neurotoxicity and neutropenia [1-3,5], whereas hypersensitivity reactions have been recognized as problematic with the increasing use of L-OHP in clinical practice [6-13]. Hypersensitivity reactions are an important adverse effect that may determine whether the treatment can be continued. We previously demonstrated that L-OHP-related grade $3 / 4$ hypersensitivity reactions occurred immediately after the initiation of infusion, whereas grade $1 / 2$ reactions did not [14]. A total of 20 laboratory test data obtained prior to the treatments were analyzed [15]. A lower serum level of lactate dehydrogenase $(\mathrm{LDH})$ was found to be a risk factor for grade $1 / 2$ reactions, while a lower monocyte count was a risk factor for grade $3 / 4$ reactions [15]. These pretreatment markers may contribute to the better management of L-OHP-induced hypersensitivity reactions; however, the treatment has to be repeated several times if it is to be tolerated by patients. The cycle number, i.e., number of repetitions, averaged $7.1 \pm 4.2( \pm S D$, range: $1-19)$ in these studies. Laboratory test data were continuously obtained to assess the condition of the patient before and after each cycle of the treatment. These values often fluctuate and are used for decision-making regarding the postponement of the next cycle of the treatment. A more detailed assessment of laboratory test data from the first to last cycle of the treatment may provide a better predictor of hypersensitivity reactions, and an additional analysis was performed herein.

\section{Methods}

Laboratory test data were obtained from 108 patients treated with the FOLFOX4 and/or mFOLFOX6 regimens at either of the Labor Health and Welfare Organization Kobe Rosai Hospital, National Hospital Organization Kobe Medical Center, Kobe University Hospital, Kobe Red Cross Hospital, or Shinko Hospital, Japan, between April 2005 and March 2009. All patients had histologically or cytologically confirmed advanced or metastatic colorectal adenocarcinoma. Patients had received no prior chemotherapy or only one regimen with a washout period of more than 4 weeks after the final day of the previous treatment. Adjuvant chemotherapy performed more than 6 months previously was not counted as previous treatment. Further eligibility criteria included: 1) age of 20-75 years; 2) Eastern Cooperative Oncology
Group (ECOG) performance status of 0 or 1 ; 3) life expectancy of 3 months or more; 4 ) adequate hematological (leukocyte count: $4,000 / \mathrm{mm}^{3}-12,000 / \mathrm{mm}^{3}$, neutrophil count: $2,000 / \mathrm{mm}^{3}$ or more, platelets: $100,000 / \mathrm{mm}^{3}$ or more), hepatic (transaminases: 2.5 times or less the upper limit of normal, total bilirubin: $2.0 \mathrm{mg} / \mathrm{dL}$ or less), and renal (serum creatinine: less than the upper limit of normal) function; and 5) the ability to take oral medication. Depending on the clinical situation, patients who did not meet the criteria could be treated with L-OHP under the careful supervision of medical doctors. Patients were excluded if they had either brain metastases, a history of other neoplasms (except for cured nonmelanoma skin carcinoma or cured carcinoma in situ), a history of severe drug allergies, interstitial pneumonitis or pulmonary fibrosis, severe pleural effusion or ascites, active infection, bowel obstruction, diarrhea, and serious uncontrolled comorbidity or medical conditions. Pregnant or lactating women or women not using effective contraception were also excluded.

Hypersensitivity reactions were assessed and classified according to the National Cancer Institute Common Criteria (NCI-CTCAE v3.0). A total of 20 laboratory test data were compared between patients who exhibited hypersensitivity reactions and those who did not, including hematological parameters (erythrocyte count, hemoglobin, hematocrit, leukocyte count, neutrophil count, lymphocyte count, eosinophil count, basophil count, monocyte count, and platelet count), hepatic parameters (aspartate aminotransferase, alanine aminotransferase, gamma-glutamyl transpeptidase, total bilirubin, and alkaline phosphatase), serum LDH, renal parameters (blood urea nitrogen and serum creatinine), carcinoembryonic antigen, and CA19-9 antigen. This retrospective study was approved by the Institutional Review Board of each of the 5 hospitals. It should be noted that laboratory test data obtained prior to the first cycle of the treatment were analyzed in terms of susceptibility to hypersensitivity reactions [15]. In this study, the time course from the first to last cycle of the treatment was additionally analyzed to identify a better predictor of hypersensitivity reactions. Laboratory test data at the $\mathrm{n}$-th cycle represented those from initiation of the $n$-th cycle to just before the initiation of $(n+1)$-th cycle of the treatment, and worst ones were adopted when they were measured more than 2 times. The averages and variation coefficients $(\mathrm{CV} \%)$ of the data until the last cycle of the treatment were used for comparisons. Fisher's exact test was used for analysis of the contingency table. The unpaired Student's $t$-test/Welch's test or Mann-Whitney's $U$ test was used for group comparisons, and after a Bonfer- 
roni correction, $\mathrm{P}$ values of less than 0.05 were considered significant.

\section{Results}

The cycle number in patients with no, grade $1 / 2$, and $3 / 4$ hypersensitivity reactions is summarized in Table 1. No significant differences was observed among 3 groups ( $p=0.149$, Fisher's). The cycle number at which grade $1 / 2$ reactions occurred was $7.5 \pm 4.6$ (range: 2-17), whereas it was $9.3 \pm 3.9$ (range: $5-16$ ) for grade $3 / 4$ reactions. An additional cycle of the treatment was not performed when grade $3 / 4$ reactions occurred, whereas it was possible for grade $1 / 2$ reactions. The average values of hematological parameters and serum LDH levels are summarized in Table 2. Serum LDH levels were lower in patients with grade $1 / 2$ reactions than in those with no reactions $(\mathrm{P}=0.016$, Mann-Whitney's); however, no significant differences were observed in hematological parameters between patients with no and grade $1 / 2$ reactions, or between those with no and grade $3 / 4$ reactions. No significant differences were noted in the other parameters examined in this study between patients (data not shown).

Laboratory test data fluctuated extensively from the first to last cycle of the treatment. CV\% values are summarized in Table 3 . Among the 20 laboratory test data analyzed herein, the $\mathrm{CV} \%$ value was the highest for the basophil count, followed by the eosinophil count, and neutrophil count. The CV\% value of serum $\mathrm{LDH}$ levels was relatively low, and a correlation was observed with hypersensitivity reactions; the CV\% value was higher in patients with grade $3 / 4$ reactions than in those with no reactions $(\mathrm{P}=0.005$, Mann-Whitney's). The difference between the maximum and minimum values was also higher in patients with grade $3 / 4$ reactions $(\mathrm{P}=0.017$, Mann-Whitney's), while no such relationship was found for the maximum values $(P=0.060$,
Mann-Whitney's). The time courses of serum LDH levels in patients with grade $3 / 4$ are shown in Figure 1 , and are from all 10 patients and expressed as a function of the cycle number. Serum LDH levels were increased in 4 of 10 patients as the cycle number increased until grade $3 / 4$ reactions manifested, and these phenomena reflected the higher value of $\mathrm{CV} \%$ in Table 3. An increase in serum LDH levels before the manifestation of hypersensitivity reactions was not observed for grade $1 / 2$ reactions.

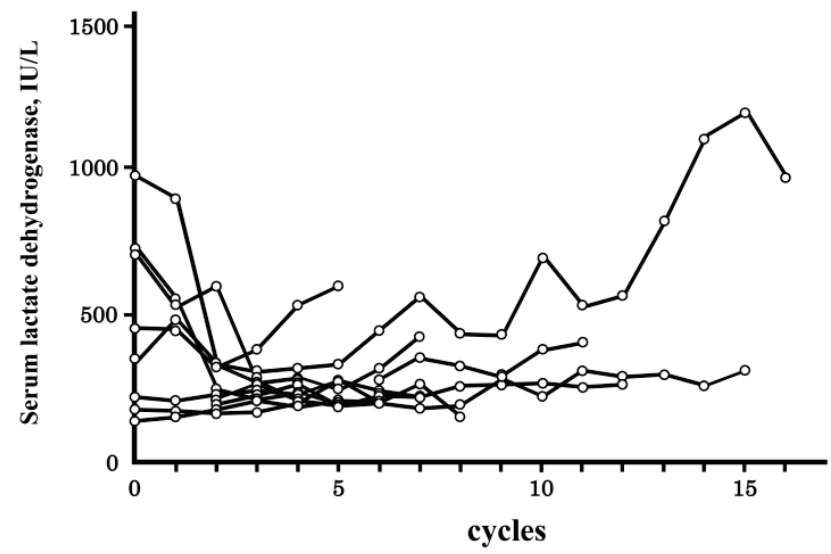

Figure I. Time courses of serum lactate dehydrogenase levels in patients with grade 3/4 hypersensitivity reactions. Time courses are indicated as a function of the cycle number, i.e., the number of treatment repetitions. Grade 3/4 hypersensitivity reactions occurred in 10 of 108 patients treated with oxaliplatin, and the time courses of all 10 patients were indicated. The cycle number at which grade $3 / 4$ hypersensitivity reactions occurred was $9.3 \pm 3.9( \pm \mathrm{SD})$, and an additional cycle of the treatment was not performed.

Table I. Cycle number of therapy in patients with no, grade I/2, and grade 3/4 hypersensitivity reactions

\begin{tabular}{lllll}
\hline & $1-5$ & $6-10$ & $11-15$ & $16-20$ \\
\hline No hypersensitivity & 38 & 31 & 13 & 2 \\
Grade $1 / 2$ hypersensitivity & 4 & 6 & 3 & 1 \\
Grade 3/4 hypersensitivity & 1 & 5 & 3 & 1 \\
\hline No significant differences were observed among the 3 groups (p=0.149, Fisher's).
\end{tabular}

Table 2. Average values of hematological parameters and serum lactate dehydrogenase levels in patients with no, grade $1 / 2$, and grade $3 / 4$ hypersensitivity reactions

\begin{tabular}{|c|c|c|c|}
\hline & $\begin{array}{l}\text { No hypersensitivity } \\
N=84\end{array}$ & $\begin{array}{l}\text { Grade } 1 / 2 \\
N=14\end{array}$ & $\begin{array}{l}\text { Grade } 3 / 4 \\
N=10\end{array}$ \\
\hline Erythrocyte count, $\times 10^{4} / \mathrm{mm}^{3}$ & $372 \pm 52[380,37]$ & $382 \pm 58[392,27]$ & $398 \pm 45[397,17]$ \\
\hline Hemoglobin, g/dL & $11.3 \pm 1.8[11.4,1.3]$ & $11.2 \pm 1.5[11.1,0.7]$ & $11.7 \pm 1.8[11.6,1.3]$ \\
\hline Hematocrit, $\%$ & $34.3 \pm 5.1[34.4,3.5]$ & $34.4 \pm 3.9[34.6,2.5]$ & $35.9 \pm 4.2[35.7,2.4]$ \\
\hline Leukocyte count, / $\mathrm{mm}^{3}$ & $5039 \pm 3036[4469,834]$ & $4457 \pm 1164[4327,775]$ & $4877 \pm 1227[4543,324]$ \\
\hline Neutrophil count, / $\mathrm{mm}^{3}$ & $3082 \pm 3051[2487,672]$ & $2325 \pm 1068[2115,869]$ & $3056 \pm 1691[2676,357]$ \\
\hline Lymphocyte count, $/ \mathrm{mm}^{3}$ & $1311 \pm 460[1322,336]$ & $1450 \pm 390[1424,171]$ & $1319 \pm 371[1364,187]$ \\
\hline Eosinophil count, $\%$ & $3.7 \pm 3.0[3.0,1.7]$ & $6.0 \pm 5.5[4.6,1.7]$ & $3.2 \pm 1.9[2.6,1.5]$ \\
\hline Basophil count, \% & $0.7 \pm 0.5[0.7,0.3]$ & $0.9 \pm 0.5[0.8,0.2]$ & $0.8 \pm 0.5[0.7,0.2]$ \\
\hline Monocyte count \% & $7.2 \pm 3.0[7.2,2.2]$ & $6.8 \pm 2.7[8.0,1.6]$ & $7.4 \pm 2.1[7.7,0.9]$ \\
\hline Platelet count, $\times 10^{4} / \mathrm{mm}^{3}$ & $20.0 \pm 7.2[19.3,4.7]$ & $17.4 \pm 6.0[16.1,2.7]$ & $18.8 \pm 8.8[15.3,3.1]$ \\
\hline Lactate dehydrogenase, IU/L & $326 \pm 266[240,80]$ & $241 \pm 172[196,22]$ * & $336 \pm 132[314,79]$ \\
\hline
\end{tabular}

Values are the means \pm standard deviations with the medians and quartile deviations in parentheses.

* significantly different from patients without hypersensitivity reactions 
Table 3. CV\% values of hematological parameters and serum lactate dehydrogenase levels in patients with no, grade $\mathrm{I} / 2$, and grade $3 / 4$ hypersensitivity reactions

\begin{tabular}{llll}
\hline & No hypersensitivity & Grade 1/2 & Grade 3/4 \\
& $\mathrm{N}=84$ & $\mathrm{~N}=14$ & $\mathrm{~N}=10$ \\
\hline Erythrocyte count , \% & $7.8 \pm 5.9[6.2,2.3]$ & $8.0 \pm 7.7[6.2,1.1]$ & $7.6 \pm 6.1[6.3,1.0]$ \\
Hemoglobin, \% & $7.6 \pm 6.1[6.0,2.6]$ & $8.1 \pm 8.1[5.5,1.4]$ & $7.4 \pm 6.0[6.1,0.7]$ \\
Hematocrit, \% & $7.6 \pm 6.1[6.5,2.8]$ & $7.8 \pm 7.7[4.9,1.6]$ & $7.2 \pm 5.6[6.0,0.4]$ \\
Leukocyte count , \% & $31.6 \pm 18.5[28.3,10.2]$ & $33.9 \pm 15.0[31.1,6.0]$ & $32.9 \pm 9.0[34.9,6.6]$ \\
Neutrophil count, \% & $48.6 \pm 24.5[45.8,14.4]$ & $52.9 \pm 25.6[54.1,13.0]$ & $55.2 \pm 28.7[54.3,13.6]$ \\
Lymphocyte count , \% & $25.8 \pm 15.4[24.3,10.2]$ & $21.4 \pm 7.3[21.4,4.9]$ & $27.2 \pm 6.3[27.4,3.1]$ \\
Eosinophil count, \% & $52.0 \pm 23.8[54.3,17.3]$ & $64.9 \pm 33.3[67.3,21.9]$ & $47.2 \pm 21.4[39.0,17.9]$ \\
Basophil count, \% & $78.5 \pm 59.0[68.9,32.6]$ & $74.0 \pm 52.9[63.1,18.9]$ & $109.5 \pm 71.2[87.9,23.0]$ \\
Monocyte count, \% & $44.2 \pm 29.0[41.0,18.6]$ & $51.2 \pm 32.0[46.1,13.1]$ & $36.2 \pm 10.9[35.7,6.9]$ \\
Platelet count, \% & $31.1 \pm 15.9[29.3,10.6]$ & $27.6 \pm 13.7[26.5,9.3]$ & $31.4 \pm 8.7[29.8,5.1]$ \\
Lactate dehydrogenase , \% & $17.8 \pm 19.3[16.4,8.0]$ & $14.8 \pm 12.6[11.7,3.6]$ & $32.5 \pm 20.5[22.4,15.3]{ }^{*}$ \\
\hline
\end{tabular}

Values are the means \pm standard deviations with the medians and quartile deviations in parentheses.

* significantly different from patients without hypersensitivity reactions

\section{Discussion}

Glycolysis, the transformation of glucose to pyruvate, is a key step for energy acquisition in all mammalian cells, including cancer cells. Pyruvate is further transformed to acetyl-CoA under normoxic conditions; however, suboptimal oxygen availability switches the metabolic pathway, resulting in the transformation to lactate via LDH-5, one of the 5 isoenzymes of LDH [16]. Immunochemical studies have shown that LDH-5 is overexpressed in non-small-cell lung cancer [17] and advanced metastatic colorectal cancer $[18,19]$. Removal of the primary tumor was shown to result in a significant reduction in serum LDH levels in patients with non-small cell lung cancer [17]; however, serum LDH levels have not always reflected the presence of a tumor [20]. In spite of the lack of information on the relationships between serum LDH levels and presence of tumors, serum LDH levels have been investigated in terms of the response to treatment and prognosis of cancer patients [17, 21-25], and higher serum levels were concluded to be associated with a poorer prognosis. We previously demonstrated that a lower serum LDH level prior to the treatments was a risk factor for grade $1 / 2$ reactions [15]. In this study, we analyzed laboratory test data from the first to last cycle of the treatment, and serum LDH levels were shown to be maintained at lower levels in patients with grade $1 / 2$ reactions than those with no reactions (Tables 2 and 3). Our results suggest that patients without the exacerbation of tumors are susceptible to grade $1 / 2$ reactions.

In our previous study, a lower monocyte count prior to the treatments was identified as a risk factor for grade 3/4 reactions [15]; however, no significant differences were observed in the average values between patients with no and grade $3 / 4$ reactions (Table 2 ). In contrast, patients with grade $3 / 4$ reactions could be characterized by larger variations in serum LDH levels (Table 3). As shown in Figure 1, serum LDH levels increased as the cycle number increased in some patients, and thereafter grade $3 / 4$ reactions occurred (Figure 1). This phenomenon was not observed in patients with grade $1 / 2$ reactions. If an increase in serum LDH levels suggests the exacerbation of a tumor, it is accompanied by grade $3 / 4$ reactions.

Hypersensitivity reactions are a well-established complication of platinum agents; however, the mechanisms responsible remain unclear [26-29]. Reactions are thought to be mainly caused by the type I IgE-mediated system because they occur after multiple infusions; however, recent studies have suggested the contribution of the type IV T-cell-mediated system [26-29]. Regarding L-OHP, most reactions are thought to be of type I, while reports of hemolysis and thrombocytopenia suggest a type II reaction, and chronic urticaria, joint pain, and proteinuria can be attributed to a type III reaction [26-29]. In the present study, the $\mathrm{CV} \%$ values of the eosinophil count were slightly higher in patients with grade $1 / 2$ reactions $(p=0.082)$, whereas those of the basophil count were higher for grade $3 / 4$ reactions $(p=0.089)$. Eosinophils and basophils are known to be involved in allergic reactions [30]. Eosinophils and basophils express many of the same receptors, whereas the cytokines produced differ [30]. The eosinophil and basophil counts may be predictive markers of L-OHP-induced hypersensitivity reactions.

In conclusion, a total of 20 laboratory test data were obtained from patients with advanced colorectal cancer who were treated with the L-OHP-containing regimens, FOLFOX4 and/or mFOLFOX6. The time course until the last cycle of the treatment was compared between patients with hypersensitivity reactions and those without. We demonstrated that the average value of serum LDH levels was lower in patients with grade $1 / 2$ reactions, whereas its $C V \%$ value was higher in patients with grade $3 / 4$ reactions 
than in those without reactions. These results suggested that the serum LDH levels may be a predictive marker of L-OHP-induced hypersensitivity reactions.

\section{Competing Interests}

The authors have declared that no competing interest exists.

\section{References}

1. de Gramont A, Figer A, Seymour M, et al. Leucovorin and fluorouracil with or without oxaliplatin as first-line treatment in advanced colorectal cancer. J Clin Oncol. 2000; 18: 2938-2947.

2. Tournigand C, André T, Achille E, et al. FOLFIRI followed by FOLFOX6 or the reverse sequence in advanced colorectal cancer: A randomized GERCOR study. J Clin Oncol. 2004; 22: 229-237.

3. Goldberg RM, Sargent DJ, Morton RF, et al. A randomized controlled trial of fluorouracil plus leucovorin, irinotecan, and oxaliplatin combinations in patients with previously untreated metastatic colorectal cancer. J Clin Oncol. 2004; 22:23-30.

4. Grothey A, Sargent D, Goldberg RM, et al. Survival of patients with advanced colorectal cancer improves with the availability of fluorouracil-leucovorin, irinotecan, and oxaliplatin in the course of treatment. J Clin Oncol. 2004; 22: 1209-1214.

5. Venook A. Critical evaluation of current treatments in metastatic colorectal cancer. Oncologist. 2005; 10: 250-261.

6. André $\mathrm{T}$, Boni $\mathrm{C}$, Mounedji-Boudiaf $\mathrm{L}$, et al. Oxaliplatin, Fluorouracil, and Leucovorin as Adjuvant Treatment for Colon Cancer. N Engl J Med. 2004; 350: 2343-2351.

7. André T, Boni C, Navarro M, et al. Improved overall survival with oxaliplatin fluorouracil, and leucovorin as adjuvant treatment in stage II or III colon cancer in the MOSAIC trial. J Clin Oncol. 2009; 27: 3109-3116.

8. Thomas RR, Quinn MG, Schuler B, et al. Hypersensitivity and idiosyncratic reactions to oxaliplatin. Cancer. 2003; 97: 2301-2307.

9. Siu SW, Chan RT, Au GK, et al. Hypersensitivity reactions to oxaliplatin: experience in a single institute. Ann Oncol. 2006; 17: 259-261.

10. Maindrault-Goebel F, Andre T, Tournigand C, et al. Allergic-type reactions to oxaliplatin: retrospective analysis of 42 patients. Eur J Cancer. 2005; 41: 2262-2267.

11. Gowda A, Goel R, Berdzik J, et al. Hypersensitivity reactions to oxaliplatin: incidence and management. Oncology. 2004; 18: 1671-1675.

12. Brandi G, Pantaleo MA, Galli C, et al. Hypersensitivity reactions related to oxaliplatin (OHP). Br J Cancer. 2003; 89: 477-481.

13. Shibata $\mathrm{Y}$, Ariyama H, Baba E, et al. Oxaliplatin-induced allergic reaction in patients with colorectal cancer in Japan. Int J Clin Oncol. 2009; 14: 397-401.

14. Seki K, Senzaki K, Tsuduki Y, et al. Multicenter trial on hyper-sensitivity reactions following treatment with FOLFOX regimens. Jpn J Pharm Health Care Sci. 2008; 34: 919-926.

15. Seki K, Senzaki K, Tsuduki Y, et al. Risk Factors for Oxaliplatin-Induced Hypersensitivity Reactions in Japanese Patients with Advanced Colorectal Cancer. Int J Med Sci. 2011; 8: 210-215.

16. Colgan SM, Mukherjee S, Major P, et al. Hypoxia-induced lactate dehydrogenase expression and tumor angiogenesis. Clin.Colorectal.Cancer. 2007; 6: $442-446$.

17. Koukourakis MI, Giatromanolaki A, Sivridis E, et al. Tumour and Angiogenesis Research Group: Lactate dehydrogenase-5 (LDH-5) overexpression in non-small-cell lung cancer tissues is linked to tumour hypoxia, angiogenic factor production and poor prognosis. Br J Cancer. 2003; 89: 877-885.

18. Koukourakis MI, Giatromanolaki A, Simopoulos C, et al. Lactate dehydrogenase 5 (LDH5) relates to up-regulated hypoxia inducible factor pathway and metastasis in colorectal cancer. Clin.Exp.Metastasis. 2005; 22: 25-30.

19. Koukourakis MI, Giatromanolaki A, Harris AL, et al. Comparison of metabolic pathways between cancer cells and stromal cells in colorectal carcinomas: a metabolic survival role for tumor-associated stroma. Cancer Res. 2006; 66: 632-637.

20. Koukourakis MI, Giatromanolaki A, Sivridis E, et al. Lactate dehydrogenase 5 expression in operable colorectal cancer: strong association with survival and activated vascular endothelial growth factor pathway--a report of the Tumour Angiogenesis Research Group. J.Clin.Oncol. 2006; 24: 4301-4308.

21. Hersey P, Watts RN, Zhang XD, et al. Metabolic approaches to treatment of melanoma. Clin.Cancer Res. 2009; 15: 6490-6494.

22. Sturgeon CM, Duffy MJ, Stenman UH, et al. National Academy of Clinical Biochemistry laboratory medicine practice guidelines for use of tumor markers in testicular, prostate, colorectal, breast, and ovarian cancers. Clin.Chem. 2008; 54: e11-79.

23. Colovic N, Tomin D, Vidovic A, et al. Pretreatment prognostic factors for overall survival in primary resistant acute myeloid leukemia. Biomed Pharmacother. 2012; 66: 578-582.

24. Culine S. Prognostic factors in unknown primary cancer. Semin.Oncol. 2009; 36: 60-64.
25. Piccaluga PP, Agostinelli C, Gazzola A, et al. Prognostic markers in peripheral T-cell lymphoma. Curr.Hematol.Malig.Rep. 2010; 5: 222-228.

26. Syrigou E, Syrigos K, Saif MW. Hypersensitivity reactions to oxaliplatin and other antineoplastic agents. Curr Allergy Asthma Rep. 2008; 8: 56-62.

27. Eng C. Toxic effects and their management: daily clinical challenges in the treatment of colorectal cancer. Nat Rev Clin Oncol. 2009; 6: 207-218.

28. Makrilia N, Syrigou E, Kaklamanos I, et al. Hypersensitivity reactions associated with platinum antineoplastic agents: a systematic review. Met Based Drugs. 2010; pii:20708

29. Lee C, Gianos M, Klaustermeyer WB. Diagnosis and management of hypersensitivity reactions related to common cancer chemotherapy agents. Ann Allergy Asthma Immunol. 2009; 102: 179-187.

30. Stone KD, Prussin C, Metcalfe DD. IgE, Mast Cells, Basophils, and Eosinophils. J Allergy Clin Immunol. 2010; 125: 73-80. 